\title{
Screening of Protein Crystallization Conditions on a Microfluidic Chip Using Nanoliter-Size Droplets
}

\author{
Bo Zheng, L. Spencer Roach, and Rustem F. Ismagilov* \\ Department of Chemistry, The University of Chicago, 5735 South Ellis Avenue, Chicago, Illinois 60637
}

Received July 9, 2003; E-mail: r-ismagilov@uchicago.edu

This paper describes a microfluidic system for screening hundreds of protein crystallization conditions using less than $4 \mathrm{~nL}$ of protein solution for each crystallization trial. Crystallization trials were set up inside 7.5-nL aqueous droplets. These droplets, each containing solutions of protein, precipitants, and additives in variable ratios, were formed in the flow of immiscible fluids inside microfluidic channels. ${ }^{1,2}$ We have used the system to set up hundreds of trials at a rate of several trials per second under computer control. The goal of this Communication is to quantify this approach and validate it by crystallizing correct polymorphs of several common watersoluble proteins.

New methods of protein crystallization are becoming especially important because of the success of genome sequencing projects. Crystallization is a bottleneck in determining tertiary protein structures from sequence data. ${ }^{3}$ Protein crystallization occurs in the labile region of the crystallization phase diagram, a narrow region where nucleation but not precipitation can occur. ${ }^{4}$ The phase diagram is multidimensional and complex, and, despite progress in theory, ${ }^{5}$ concentrations of the protein and the reagents (precipitants, buffers, and additives) that place the solution into the labile region are usually determined by screening. Minimal volumes of the protein solution should be used during screening, because many proteins are only available in very small quantities. ${ }^{6}$

Manual screening by mixing stock solutions in many ratios is time-consuming and requires at least $100 \mathrm{~nL}$ of the protein solution per trial. To overcome these limitations, robotic systems have been developed that can perform automated mixing of stock solutions, and which can set up crystallization trials with volumes from $1 \mu \mathrm{L}$ down to $100 \mathrm{~nL},{ }^{7}$ consuming as little as $10 \mathrm{~nL}$ of individual solutions. ${ }^{8}$ These robotic systems are expensive and have not yet seen wide adoption in individual laboratories.

Microfluidic systems are useful for experiments that require minimal use of reagents. ${ }^{9}$ Microfluidic platforms, therefore, are an attractive choice for macromolecular crystallization, ${ }^{6}$ as was clearly demonstrated by Hansen et al. ${ }^{10}$ These authors have crystallized proteins on a microfluidic device by free interface diffusion, a method that was previously possible only in microgravity. ${ }^{10}$ Only $\sim 10 \mathrm{~nL}$ of the protein solution was used for each of 144 trials, which were conducted inside microfabricated chambers controlled by pressure-operated valves.

The system described here (Figure 1) used three steps to crystallize proteins inside droplets, implemented using PDMS microfluidic devices with channels of $150 \times 100 \mu \mathrm{m}^{2}$ crosssectional dimensions: ${ }^{1,2}$ (1) Aqueous stock solutions were loaded into syringes, and syringes were connected to the convening channels of a microfluidic device. Only one stock solution and one syringe were required for each reagent or protein. A syringe containing water-immiscible fluorinated oil was connected to a perpendicular channel. (2) The flow of the aqueous solutions and the oil was established by driving syringes with syringe pumps. Aqueous solutions combined in the narrow droplet-forming region
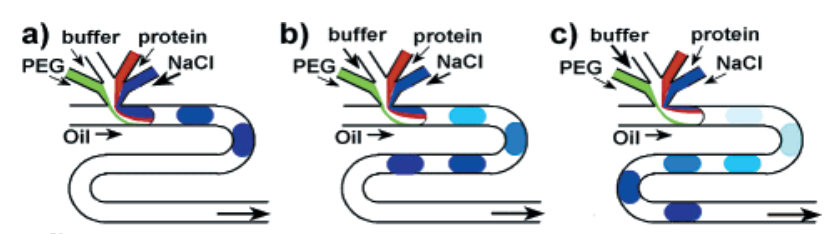

d)
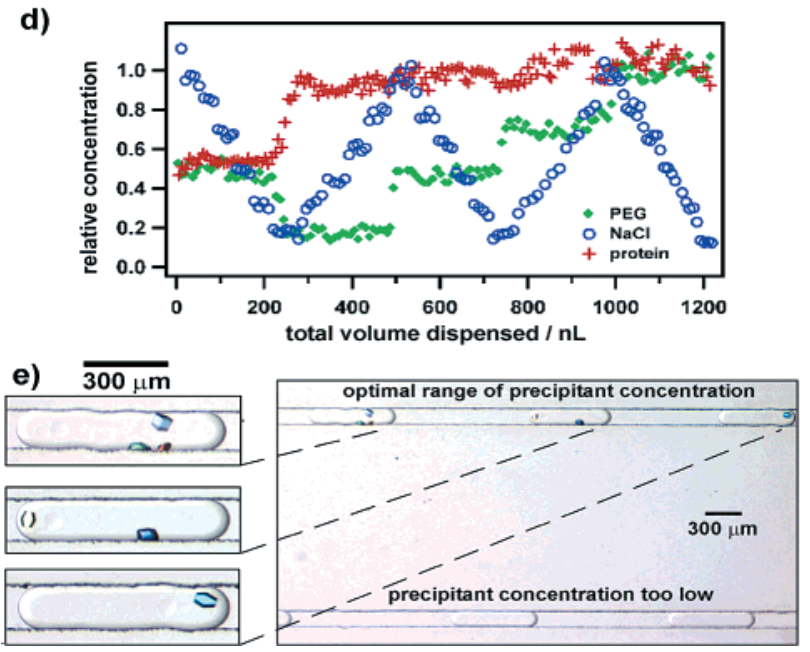

Figure 1. Droplet-based microfluidic system for protein crystallization. $(a-c)$ A schematic illustration: as the flow rate of the $\mathrm{NaCl}$ stream is decreased and the flow rate of the buffer stream is increased, the volume of the $\mathrm{NaCl}$ solution injected into each droplet decreases, and the concentration of $\mathrm{NaCl}$ in each droplet decreases. The shade of the droplets represents $\mathrm{NaCl}$ concentration. Each successive droplet represents a trial that tests a different ratio of stock solutions. (d) Experimental characterization of the idea shown in $(\mathrm{a}-\mathrm{c})$. Flow rate of oil (a 10:1 mixture of $\mathrm{C}_{14} \mathrm{~F}_{12}$ and $\left.\mathrm{C}_{6} \mathrm{~F}_{13}\left(\mathrm{CH}_{2}\right)_{2} \mathrm{OH}\right)$ was kept at $12 \mathrm{~nL} \mathrm{~s}^{-1}$. Flow rates of aqueous solutions of PEG, salt, lysozyme, and acetate buffer were varied between 0.8 and 5 $\mathrm{nL} \mathrm{s}^{-1}$ over the course of the program. The total flow rate of the aqueous solutions was kept constant at $15 \mathrm{~nL} \mathrm{~s}^{-1}$. Two 7.5-nL droplets were formed per second. Each data point represents a single droplet. (e) A polarized microphotograph illustrating crystallization of lysozyme inside droplets of variable composition on a microfluidic chip.

and entered the flowing stream of oil, where they spontaneously formed droplets; no valves were required to meter the droplets in this system. The volume of each aqueous solution injected into a droplet is directly proportional to the volumetric flow rate of that solution at the time when the droplet formed. Therefore, the ratio of volumes of the aqueous solutions injected into each droplet can be varied by varying the flow rates continuously and rapidly on the time scale of droplet formation, so each droplet has a different composition (Figure $1 \mathrm{a}-\mathrm{c}$ ). This approach is different from the one we used in kinetic measurements, ${ }^{2}$ where sets of hundreds of identical droplets were formed to perform each kinetic measurement. (3) After the last droplet was formed, the syringes were disconnected, and the flow was stopped. The device was incubated at constant temperature and monitored periodically to detect formation of crystals. 


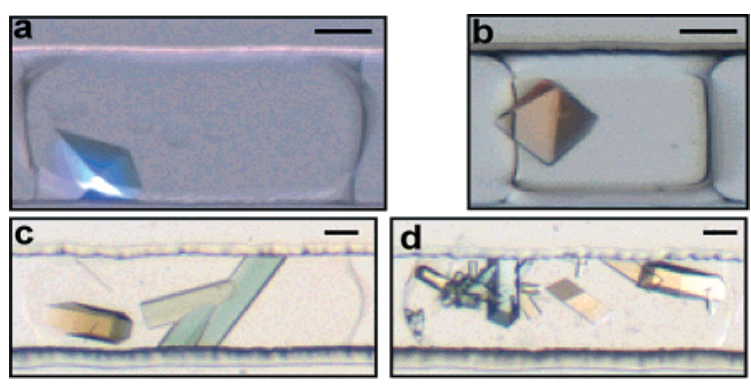

Figure 2. Polarized light micrographs of protein crystals obtained inside droplets on a microfluidic chip. Each scale bar is $50 \mu \mathrm{m}$. (a, b) Thaumatin, (c) bovine liver catalase, and (d) glucose isomerase. The crystal in (b) was obtained by allowing partial evaporation of droplets.

Precise handling of viscous solutions is challenging in a microfluidic device; in addition, concentrated solutions of proteins may affect surface tensions in the system and prevent reliable formation of droplets. Therefore, we quantified this method using stock solutions of $\mathrm{NaCl}(1.0 \mathrm{M}$, viscosity $1 \mathrm{cP})$, poly(ethylene glycol) (PEG, 30\% w/v aqueous PEG 6000, viscosity $16 \mathrm{cP}),{ }^{11}$ and a concentrated solution of lysozyme $(100 \mathrm{mg} / \mathrm{mL})$ (Figure $1 \mathrm{~d})$. We performed three experiments that were identical, except in each experiment one of the three stock solutions was labeled with fluorescein. The flow rates were varied according to the computer program, designed to vary the concentration of $\mathrm{NaCl}$ continuously for each of the combinations of four concentrations of PEG and two concentrations of lysozyme. The flows were stopped, and the concentration of the reagent from the labeled stock solution was determined by measuring the fluorescence of each droplet. The concentrations followed the program to $\sim 15 \%$, although some noise in Figure 1d is due to errors in the fluorescent measurements.

Crystallization of proteins inside droplets was monitored after the syringes were disconnected and the inlets were sealed, while channels remained in contact with the oil reservoir. Devices were incubated in a Petri dish in contact with water, which served to prevent evaporation of water from the droplets through PDMS. Droplets were then monitored periodically under a microscope. A program similar to the one described in Figure 1d was used for screening of crystallization conditions for lysozyme. Lysozyme crystals appeared only in areas that had the optimal range of conditions for crystallization in the droplets (Figure 1e). The optical transparency of PDMS simplified observation. ${ }^{12}$

We used this method to mimic two conventional screening techniques: microbatch, where no evaporation of the aqueous phase is allowed, and vapor diffusion, where the aqueous phase is allowed to evaporate slowly. In the absence of evaporation, we screened crystallization conditions for thaumatin (22 kDa, Figure 2a). The system behaved reproducibly in three different chips, giving crystals only in droplets containing $23 \mathrm{mg} / \mathrm{mL}$ protein in $0.05 \mathrm{M}$ pH 6.5 ADA and $0.34 \pm 0.04 \mathrm{M}$ sodium potassium tartrate $(0.03 \mathrm{M} \mathrm{pH}$ 7.5 HEPES). We confirmed that the conditions determined from the screen gave crystals in a standard microbatch setup. Similarly, using this chip, we obtained crystals of bovine liver catalase (230 $\mathrm{kDa}$ ) and glucose isomerase (173 kDa) (Figure 2c,d). As a proof of concept, by incubating the PDMS device without adding water to the Petri dish, we allowed partial evaporation of droplets containing crystallization mixtures for thaumatin (Figure 2b). Crystals formed at concentrations that, without evaporation, did not yield crystals. We are currently characterizing the rates of evaporation of water to quantify this system further by varying ambient humidity and the thickness of PDMS devices.
This system has several features that make it attractive for screening of conditions for protein crystallization. It uses only 4 $\mathrm{nL}$ of the protein solution for each trial in droplet, and has the potential to be scaled down further, because aqueous droplets with volumes ranging from $100 \mathrm{~nL}$ to $10 \mathrm{pL}$ can be reliably formed. ${ }^{1}$ This method of crystallization over a range of volumes may be useful for establishing the fundamental limits (e.g., nucleation) of miniaturization of crystallization. It is rapid and minimizes manual labor: once syringes with stock solutions are connected to the chip, hundreds of mixtures of solutions are prepared on the chip in $\sim 1-2$ min. This system is not limited to PDMS and will operate in other microchannels, the surfaces of which can be made hydrophobic (glass, plastics). It is especially suitable for optimizing crystallization conditions that are established, for example, by a sparse-matrix screen on a microfluidic chip described by Hansen et al. ${ }^{10}$ or a more traditional screen, like those from Hampton Research. Traditionally, only $2-4$ of the reagents are mixed in every trial during screening. Handling 5-10 solutions on this chip is realistic, and it presents an opportunity to perform multidimensional screening, where many $(5-10)$ solutions are mixed in every trial. Such screening would also provide multidimensional phase diagrams for protein crystallization, important for fundamental understanding of protein crystallization. This system should be applicable to crystallizing other classes of molecules.

In principle, crystals that we obtained during screening are large enough for structural determination by synchrotron radiation, and we are currently optimizing crystal harvesting and manipulation, already demonstrated in PDMS-based microfluidics by Hansen et al. ${ }^{10}$ We believe that this simple system will be used in individual research labs to answer fundamental questions in crystallization and will be used for crystallization of new targets. This system also has the potential to serve as the basis of high-throughput, automated crystallization systems.

Acknowledgment. This work was supported by the Searle Scholars Program and by the Beckman Young Investigator Program, and was performed at the MRSEC microfluidic facility funded by NSF. We thank Professors Keith Moffat, Phoebe Rice, and Chuan He for valuable suggestions, and we thank H. Song and J. Tice for experimental advice.

\section{References}

(1) Song, H.; Tice, J. D.; Ismagilov, R. F. Angew. Chem., Int. Ed. 2003, 42, 768-772. Tice, J. D.; Song, H.; Lyon, A. D.; Ismagilov, R. F. Langmuir 2003, 19, in press.

(2) Song, H.; Ismagilov, R. F. submitted.

(3) Chayen, N. E. Trends Biotechnol. 2002, 20, 98-98.

(4) Luft, J. R.; DeTitta, G. Macromolecular Crystallography, Pt A; Academic Press: San Diego, CA, 1997; Vol. 276, pp 110-131.

(5) Oxtoby, D. W. Nature 2001, 413, 694-695.

(6) van der Woerd, M.; Ferree, D.; Pusey, M. J. Struct. Biol. 2003, 142, 180187.

(7) Rupp, B. Acc. Chem. Res. 2003, 36, 173-181

(8) Adams, M. W. W.; Dailey, H. A.; Delucas, L. J.; Luo, M.; Prestegard, J. H.; Rose, J. P.; Wang, B. C. Acc. Chem. Res. 2003, 36, 191-198.

(9) Auroux, P.-A.; Iossifidis, D.; Reyes, D. R.; Manz, A. Anal. Chem. 2002, 74, 2637-2652.

(10) Hansen, C. L.; Skordalakes, E.; Berger, J. M.; Quake, S. R. Proc. Natl. Acad. Sci. U.S.A. 2002, 99, 16531-16536.

(11) Ninni, L.; Burd, H.; Fung, W. H.; Meirelles, A. J. A. J. Chem. Eng. Data 2003, 48, 324-329.

(12) Observing crystals through curved surfaces of droplets is not a problem in this system, because we used oil $\left(n_{\mathrm{D}}=1.3348\right)$ with a refractive index similar to that of water $\left(n_{\mathrm{D}}=1.3330\right)$, and in some cases the aqueous/oil interface was even difficult to see.

JA037166V 